\title{
Post-kala-azar dermal leishmaniasis mimicking leprosy: experience with 4 patients, with some unusual features in 1
}

\author{
SANDIPAN DHAR, INDERJEET KAUR, \\ GOUTAM DAWN, SHOBHA SEHGAL* \& \\ BHUSHAN KUMAR† \\ Departments of Dermatology, Venereology and Leprology, and \\ *Immunopathology, PGIMER, Chandigarh-160012, India
}

Accepted for publication 30 January 1995

\begin{abstract}
Summary We report on 4 cases of post-kala-azar dermal leishmaniasis (PKDL). History of kala-azar was available in all 4 patients. Slit-skin smears (SSS) for leishmania donovani (LD) bodies were negative in all 4. In 3 patients hypopigmented lesions were present over the face. Papules and nodules over his lips, tongue, scrotum and dactylitis were some unusual features observed in 1 patient. Histopathological examination showed LD bodies in 2 patients; histopathology was nonspecific in the other 2. All the patients were treated with sodium stibogluconate, $20 \mathrm{mg} / \mathrm{kg} / \mathrm{day}$. Infiltrated papules and nodules had subsided by 3 months, while hypopigmented macules took longer to improve. In 3 patients there had previously been a misdiagnosis as leprosy sufferers and they had been treated with antileprosy drugs. Clinical and histopathological differences between PKDL and leprosy are discussed.
\end{abstract}

\section{Introduction}

Post-kala-azar dermal leishmaniasis (PKDL) is an unusual complication of visceral leishmaniasis (kala-azar) which is characterized by the development of hypopigmented macules, infiltrated papules, plaques and nodules over the skin. These usually occur after a variable period of treatment of kala-azar, although, occasionally, a history of kalaazar may be absent. ${ }^{1,2}$ The condition is widely prevalent in Eastern India ${ }^{3,4}$ and less frequently in the Sudan ${ }^{2,5,6}$ and Kenya. ${ }^{7}$ While in Indian cases lesions of PKDL develop $1-5$ years after the apparent cure of visceral leishmaniasis, ${ }^{3}$ those in Africa may develop during or shortly after treatment of kala-azar. ${ }^{5,7}$

Various similarities between PKDL and leprosy make differentiation difficult. Infiltrated papules, plaques and nodules over the face, earlobes and trunk are seen in both lepromatous leprosy (LL) and PKDL, whereas plaques and hypopigmented

$\dagger$ Correspondence: Department of Dermatology, Venereology and Leprology, Postgraduate Institute of Medical Education and Research, Chandigarh-160012, India. 
macules seen in PKDL often create confusion with various types of borderline leprosy. Histologically the epithelioid cell granuloma may simulate tuberculoid leprosy, particularly when the parasites are scanty and are not identified in the infiltrate. ${ }^{8}$ The occurrence of dermal nerve involvement in PKDL has further complicated the issue of differentiating PKDL from leprosy. ${ }^{9}$

The aim of the present communication is to highlight how many cases of PKDL are misdiagnosed as leprosy in an endemic area and how to differentiate both conditions by clinical examination and laboratory investigations. We also describe some uncommon features, namely dactylitis and genital involvement, observed in 1 of the 4 patients.

\section{Case reports}

CASE 1

A 20-year-old man, a migrant from Bihar, presented with asymptomatic erythematous papules, plaques and nodules over his face, neck and trunk, with hypopigmented patches on his back of 3 years' duration. He gave a history of kala-azar when he was 5 years old, for which he had received some injections. He was diagnosed as a case of lepromatous leprosy in a hospital in Bihar and was treated with multibacillary WHO multidrug therapy (WHO-MDT-MB), for 1 year, after which he stopped treatment due to a lack of response.

On general examination, he was mildly anaemic, with no jaundice or hepatosplenomegaly. Cutaneous examination revealed diffuse infiltration and erythema over his face and earlobes. There were multiple asymptomatic papules and nodules over his forehead, chin, nose, pinnae, lips and the dorsum of the tongue (Figures 1 and 2). There was a differential involvement of the central part of his face. However, there was no madarosis. Numerous lesions of similar morphology were seen over his neck, trunk and extremities, scrotum and shaft of the penis, with most of the intervening

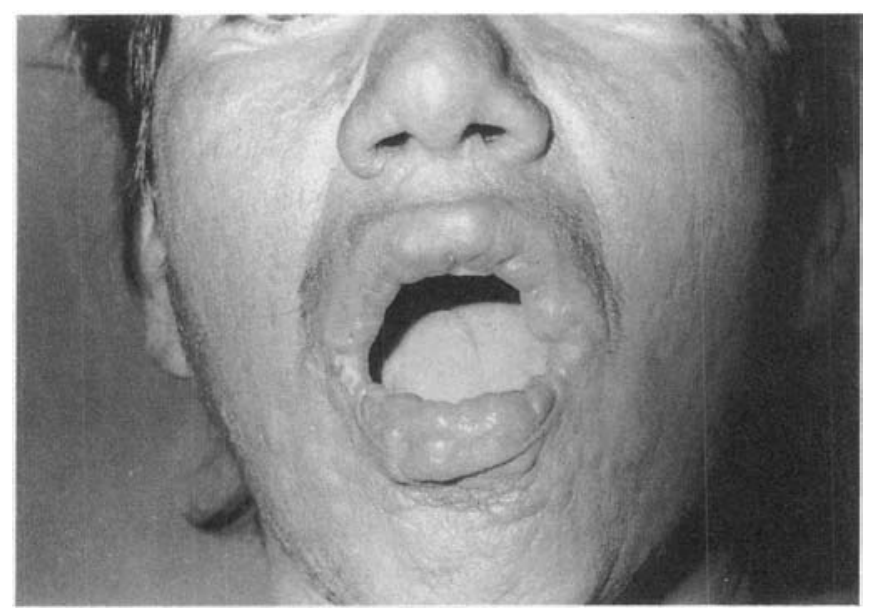

Figure 1. Showing diffuse infiltration of the face with multiple papules and nodules over cheek, chin and lips (Case 1). 


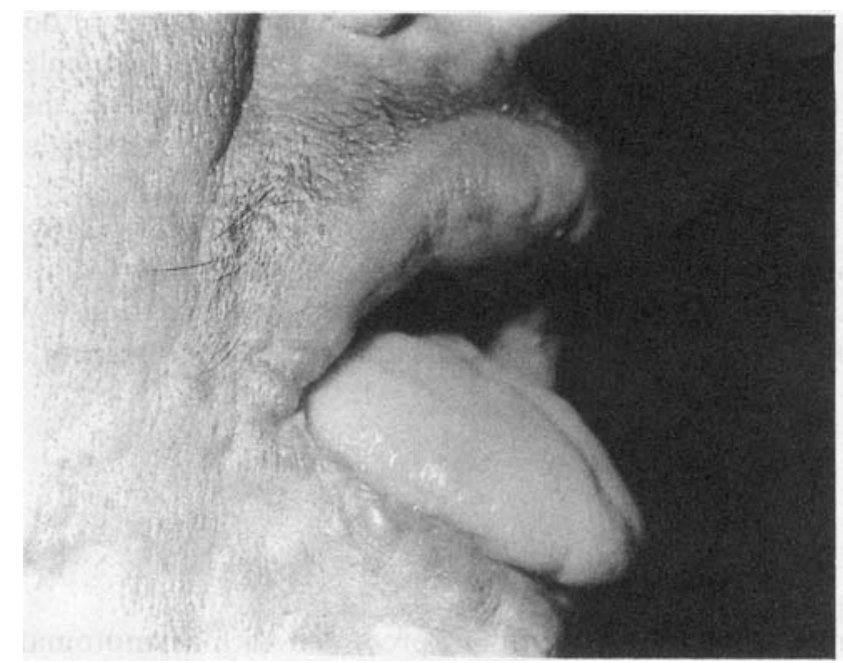

Figure 2. (Close up) Infiltrated papules and nodules over lips, lateral border and dorsum of the tongue (Case 1).

areas looking normal (Figure 3). However, the acral part of his extremities were shiny and diffusely infiltrated. There were multiple, well-defined, nonscaly, hypopigmented macules over his trunk causing no loss of sensation. Peripheral nerves were neither thickened nor tender. Some of the lesions in and around his axillae and back showed follicular keratotic plugging. In addition, he had painful fusiform swelling of the right index finger suggestive of dactylitis.

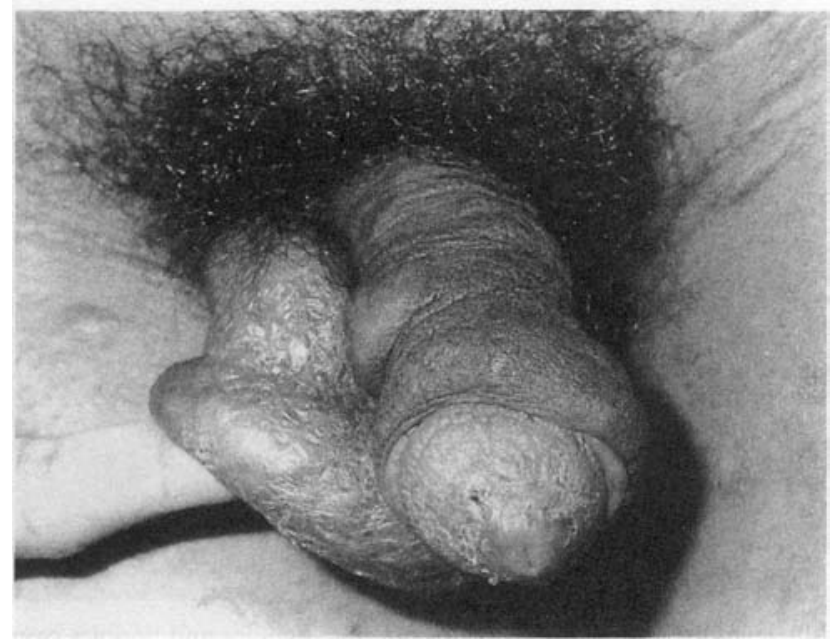

Figure 3. Erythematous papules and nodules over the scrotum, shaft of the penis and glans penis (Case 1). 
CASE 2

A 31-year-old man, a migrant from Bihar, presented with multiple erythematous papules, plaques and nodules over his earlobes, face and extremities of 6 years' duration. He had a history suggesting he had suffered from kala-azar twice before, for which he had been treated with some injections daily for 10-12 days each time. $\mathrm{He}$ was diagnosed as a case of leprosy for his current symptoms and treated with WHO-MDT-MB in a district hospital with no benefit. Examination revealed similar features to Case 1.

\section{CASE 3}

A 25-year-old woman, a migrant from Nepal, presented with multiple hypopigmented macules over her trunk and extremities which had been preceded by a generalized pruritus of 2 years' duration. She had had kala-azar 6 years before, which had been confirmed by bone marrow, and had been treated accordingly.

General examination was noncontributory. Cutaneous examination revealed multiple ill-defined, nonscaly hypopigmented macules of $0.5-1 \mathrm{~cm}$ diameter over her face, trunk and extremities. There was no loss of sensation over the macules, or any peripheral loss of sensations or nerve thickening.

\section{CASE 4}

A 41-year-old woman, a migrant from Bihar, presented with asymptomatic hypopigmented macules over her trunk and extremities of 3 years' duration. She was diagnosed as a case of leprosy and treated with WHO-MDT-MB for 2 years by a local doctor, with no advantage. A history of kala-azar was available from 10 years before, for which she had been treated with some injections given daily for 1 month.

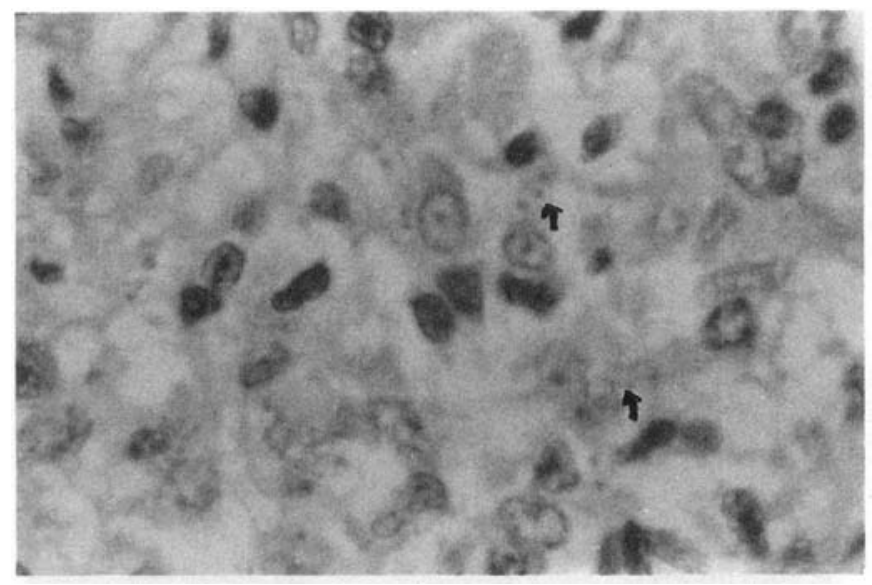

Figure 4. Showing lymphomononuclear cells in the dermis and many LD bodies (arrow) both inside and outside macrophages $(\mathrm{H} \& \mathrm{E} \times 400)($ Cases 1 and 2$)$. 
Examination revealed similar features to Case 3. She was diagnosed as a case of PKDL, although borderline lepromatous (BL) leprosy and vitiligo were other possibilities considered.

Slit-skin smears (SSS) from their earlobes, hypopigmented macules and nodular lesions ( 5 sites) in Cases 1 and 2 and from hypopigmented macules in Cases 3 and 4 were negative for acid-fast bacilli (AFB) or leishmania donovani (LD) bodies. Histopathological examination of the biopsy specimen from nodular lesions in Cases 1 and 2 and hypopigmented macules in Cases 3 and 4 showed flattened epidermis, with dermal infiltrate composed predominantly of lymphocytes and plasma cells. The infiltrate was dense in Cases 1 and 2 and sparse in Cases 3 and 4. Staining with Giemsa showed many LD bodies in Cases 1 and 2 (Figure 4), while none could be demonstrated in Cases 3 and 4. Careful histopathological examination ruled out other clinical possibilities. Except for mild anaemia, all other routine investigations on haemogram, serum biochemistry, urine, stool, skiagram chest and electrocardiogram were within normal limits.

Serum electrophoresis showed hypergammaglobulinaemia in Cases 1 and 2. However, in both cases the aldehyde test was negative and a sea water test was positive. A leishmanin skin test was negative in both these patients. Indirect immunofluorescence (IIF) test using LD bodies as substrate was positive $(1: 200)$ in Case 2 (Figure 5).

All patients were treated with intramuscular injections of sodium stibogluconate, $20 \mathrm{mg} / \mathrm{kg} / \mathrm{day}$. Infiltrated papules and nodules subsided after 1 month and 3 months respectively in Cases 1 and 2. Hypopigmented macules started fading gradually after 3 months of full treatment. There was no relapse of skin lesions in any of the 4 patients during a subsequent follow-up of more than 2 years.

\section{Discussion}

PKDL mainly occurs in India, particularly Eastern India, i.e. West Bengal, Bihar and the areas of Nepal adjoining these states. After apparent control, a recent trend of

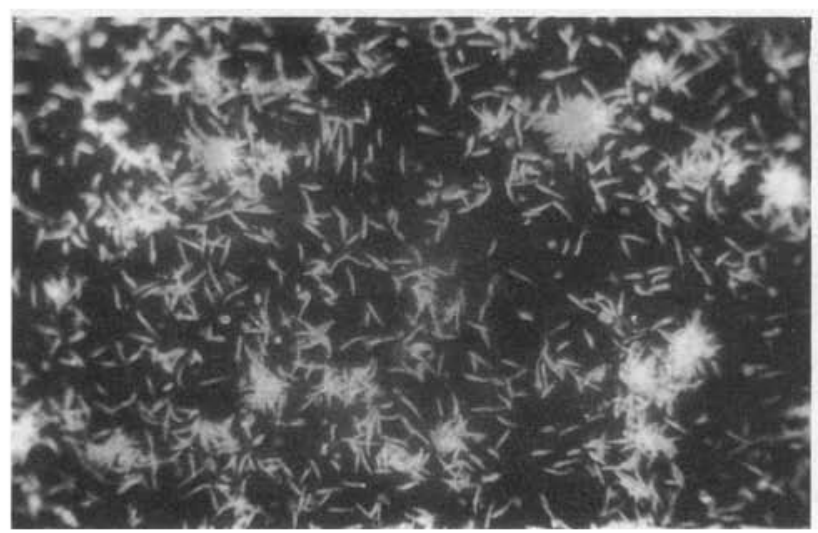

Figure 5. Positive indirect immunofluorescence (IIF) test using LD bodies as substrate (Case 2). 
increase in the incidence of PKDL has been noticed in Bihar. ${ }^{10}$ (Incidentally 3 of our patients were migrants from Bihar.) Our patients split 50:50 men:women, although a female preponderance has been noticed by others. ${ }^{11}$ A history of kala-azar was available in all the patients, as observed by other Indian workers. ${ }^{10}$ However, in African cases such a history is not always forthcoming. ${ }^{1,2}$

SSS usually yield AFB in almost all LL or BL leprosy cases as compared to PKDL where LD bodies have not been found in more than $60 \%$ of cases. ${ }^{10}$ SSS were negative for LD bodies in all our patients as has been observed earlier. ${ }^{12}$ SSS yield LD bodies mostly from infiltrated lesions, namely papules/plaques and nodules and less frequently from hypopigmented macules. 3,4

While histopathology of bacillary positive leprosy is quite characteristic, that of PKDL mostly shows nonspecific dermal infiltrate by lymphomononuclear cells. ${ }^{10}$ However, epithelioid cell granuloma occasionally found in PKDL may simulate tuberculoid leprosy. ${ }^{12}$ While AFB are found in histologic sections in almost every case of $\mathrm{LL}$ or BL, LD bodies could be demonstrated only in $50 \%$ of cases with PKDL in a large series. ${ }^{10}$ In the present report only in Cases 1 and 2 could LD bodies be demonstrated on histopathological examination. With the recent observation of perineural infiltration in PKDL, the issue of differentiating it from leprosy has been further complicated. ${ }^{9}$ However, direct and indirect immunofluorescence tests for PKDL can overcome this limitation to a great extent. ${ }^{2}$ The face is supposed to be the commonest site for hypopigmented lesions in PKDL. ${ }^{13}$ We observed such lesions in 3 of our patients over the face. Though macrocheilia has been described in PKDL by Sen Gupta, ${ }^{14}$ nodules over the lips and tongue, as seen in Case 1, are unusual. Similarly dactylitis observed in the same patient is as yet unreported in PKDL to the best of our knowledge, and the occurrence of genital lesions as seen in our patients, though known in patients with LL or BL disease, is extremely rare in PKDL.

It is evident from our patients that papules and nodules respond earlier and better to stibogluconate as compared to hypopigmented macules, which require at least 3 months to show improvement. This has been observed before. ${ }^{15}$

Cases 1, 2 and 4 were misdiagnosed as having leprosy and treated with antileprosy drugs. This is quite a common error since both leprosy and PKDL have many features in common ${ }^{16,17}$ and both diseases are prevalent in the same geographical areas. However, the absence of madarosis in the presence of facial infiltration (seen in Cases 1 and 2), intact sensations over lesions and peripheral parts, the absence of nerve thickening and tenderness are some clinical pointers towards a diagnosis of PKDL. SSS is a simple procedure which can be carried out in the outpatient department to overcome the problem in many cases.

\section{References}

${ }^{1}$ El Hassan AM, Ghalib HW, Zijlstra E, El Toum IA, Ali MS, Ahmed HMA. Post kala-azar dermal leishmaniasis in the absence of visceral leishmaniasis. Lancet, 1990; 336: 750.

2 El Hassan AM, Ghalib HW, Zijlstra E, El Toum IA, Satti M, Ali MS, Ali HMA. Post kala-azar dermal leishmaniasis in the Sudan: clinical features: pathology and treatment. Trans Roy Soc Trop Med Hyg, 1992; 86: $245-8$.

3 Napier LE, Das Gupta CR. A clinical study of post kala-azar dermal leishmaniasis. Ind Med Gaz, 1930; 65: 249-57. 
4 Thakur CP. Epidemiological, clinical and therapeutic features of Bihar kala-azar (including post kala-azar dermal leishmaniasis). Trans Roy Soc Trop Med Hyg, 1984: 78: 391-8.

5 Kirk R, Satti MH. Studies in leishmaniasis in the Anglo-Egyptian Sudan. IV. A punctate rash in treated cases. Trans Roy Soc Trop Med Hyg, 1940; 34: 213-16.

${ }^{6}$ Satti MH. Cutaneous and mucocutaneous lesions in kala-azar. Sudan Med J, 1963; 2: 88-103.

7 Rees PH, Karger PA. Visceral leishmaniasis and post kala-azar dermal leishmaniasis. In: The Leishmaniasis in Biology and Medicine, Vol. 2. Peters W, Killick-Kendrik R (eds). London: Academic Press, 1987, pp. 583615.

8 Girgla HS, Marsden RA, Singh GM, Ryan TJ. Post kala-azar dermal leishmaniasis. Br J Dermatol, 1977; 97: 307-11.

9 El Hassan AM, Ali MS, Zijlstra EE, Eltoum IA, Ghalib HW, Ahmed AMA. Post kala-azar dermal leishmaniasis in the Sudan: peripheral neural involvement. Int J Dermatol, 1992; 31: 400-3.

10 Sinha SM, Gupta G, Ghosh RN, Ganguly U, Das Gupta A, Mukherjee C. Post kala-azar dermal leishmaniasis: a clinicopathologic study. Ind J Dermatol Venereol Le prol, 1993; 59: 172-5.

11 Sharma MC, Gupta AK, Saran R. The effect of age and sex on incidence of kala-azar. J Common Dis, 1990, 22: $277-8$.

12 Sen Gupta OC, Bhattachar jee B. Histopathology of post kala-azar dermal leishmaniasis. J Trop Med Hyg, 1953; 56: 110-16.

13 Thakur CP, Kumar K. Efficacy of prolonged treatment with stibogluconate in post kala-azar dermal leishmaniasis. Ind J Med Res, 1990; 91: 144-8.

14 Sen Gupta PC. Post kala-azar dermal leishmaniasis. Bull Calcutta School Trop Med, 1957; 5: 107-10.

15 Nandy A, Neogy B, Ghosh Dastidar M, Sarkar M, Mallick KK, Chowdhury A B. Immune response and drug response in kala-azar and post kala-azar dermal leishmaniasis. Proceedings of Indo-UK Workshop on Leishmaniasis, 1983, pp. 161-70.

16 El Hassan AM, Hashim FA, Abdullah M, Zijlstra EE, Ghalib HW. Distinguishing post kala-azar dermal leishmaniasis from leprosy: experience in the Sudan. Lepr Rev, 1993; 64: 53-9.

17 Ramesh V, Saxena U, Misra RS, Mukher jee A. Post kala-azar dermal leishmaniasis from leprosy: Lepr Rev, 1991; 62: 217-21. 